\title{
Stability Theorems for Fourier Frames and Wavelet Riesz Bases
}

\author{
Radu Balan \\ Program in Applied and Computational Mathematics \\ Princeton University \\ Princeton, NJ 08544 \\ e-mail address: rvbalan@math.princeton.edu
}

January 3, 1997

\begin{abstract}
In this paper we present two applications of a Stability Theorem of Hilbert frames to nonharmonic Fourier series and wavelet Riesz basis. The first result is an enhancement of the Paley-Wiener type constant for nonharmonic series given by Duffin and Schaefer in [6] and used recently in some applications (see [3]). In the case of an orthonormal basis our estimate reduces to Kadec' optimal 1/4 result. The second application proves that a phenomenon discovered by Daubechies and Tchamitchian [4] for the orthonormal Meyer wavelet basis (stability of the Riesz basis property under small changes of the translation parameter) actually holds for a large class of wavelet Riesz bases.
\end{abstract}

Key words: Frames, Riesz basis, nonharmonic series, wavelets

Math Subject Classification: Primary $42 \mathrm{C} 15$ Secondary $41 \mathrm{~A} 30^{1}$

\footnotetext{
${ }^{1}$ published in "The Journal of Fourier Analysis and Applications", vol.3, no.5, 1997, 499-505
} 


\section{Introduction and Statement of the Results}

Let $H$ be a separable Hilbert space and $\mathbf{I}$ a countable index set. A set of vectors $\mathcal{F}=\left\{f_{i}\right\}_{i \in \mathbf{I}}$ in $H$ is called a (Hilbert) frame if there are two positive constants $0<A \leq B<\infty$ such that for all $x \in H$ we have:

$$
A\|x\|^{2} \leq \sum_{i \in \mathbf{I}}\left|<x, f_{i}>\right|^{2} \leq B\|x\|^{2}
$$

If $A=B$ we call the frame tight. The constants $A$ and $B$ are called frame bounds. A set of vectors $\mathcal{F}=\left\{f_{i}\right\}_{i \in \mathbf{I}}$ in $H$ is called Bessel set (if $\mathbf{I}=\mathbf{N}$, it is called Bessel sequence) if there exists a positive constant $B>0$ such that for any $x \in H$ we have:

$$
\sum_{i \in \mathbf{I}}\left|<x, f_{i}>\right|^{2} \leq B\|x\|^{2}
$$

If the set $\mathcal{F}$ is a (Schauder) basis as well as a frame in $H$ then $\mathcal{F}$ is called Riesz basis.

The following result is the stability theorem for Hilbert frames:

Stability Theorem for Hilbert Frames [Paley-Wiener-Kato-Christensen] Suppose H a separable complex Hilbert space, $\mathbf{I}$ a countable index set and $\mathcal{F}=\left\{f_{i}\right\}_{i \in \mathbf{I}}$ a frame in $H$ with bounds $A, B$. Consider $\mathcal{G}=\left\{g_{i}\right\}_{i \in \mathbf{I}}$ a family of vectors in $H$. If one of the following two conditions is fulfilled:

(Type 1) $\quad\left(\sum_{i \in \mathbf{I}}\left|<x, f_{i}-g_{i}>\right|^{2}\right)^{1 / 2} \leq \lambda\left(\sum_{i \in \mathbf{I}}\left|<x, f_{i}>\right|^{2}\right)^{1 / 2}+\mu\|x\|, \forall x \in H$

or:

(Type 2) $\quad\left\|\sum_{n \in I_{N}} c_{i}\left(f_{i}-g_{i}\right)\right\| \leq \lambda\left\|\sum_{n \in I_{N}} c_{i} f_{i}\right\|+\mu\left(\sum_{n \in I_{N}}\left|c_{i}\right|^{2}\right)^{1 / 2}, \forall n \geq 0, c_{i} \in \mathbf{C}$

where $\left(I_{n}\right)_{n \geq 0}$ is an increasing sequence of finite subsets of $\mathbf{I}: I_{0} \subset I_{1} \subset I_{2} \subset \ldots \subset \mathbf{I}$ such that $\lim _{n \rightarrow \infty} I_{n}=\mathbf{I}$, and $\lambda+\frac{\mu}{\sqrt{A}}<1$; then $\left\{g_{i}\right\}_{i \in \mathbf{I}}$ is also a frame in $H$ with bounds $A\left(1-\lambda-\frac{\mu}{\sqrt{A}}\right)^{2}$, $B\left(1+\lambda+\frac{\mu}{\sqrt{B}}\right)^{2}$. Moreover, if $\mathcal{F}$ is a Riesz basis then $\mathcal{G}$ is also a Riesz basis.

This result was first stated by Paley and Wiener in their celebrated paper [11]. They considered only the stability of Riesz basis and the type 2 condition. Later on, in a different context, Kato ([9]) proved a perturbation theorem which basically incorporates the above theorem. Recently, Christensen and Heil ([1], [2]) established the link between Kato's perturbation theorem and frames in both Hilbert and Banach contexts. We are going to use this result in studying the stability of two particular frames under parametric perturbations.

Consider $\gamma>0$ and $L^{2}[-\gamma, \gamma]$ with the usual scalar product inherited from $L^{2}$. Consider a sequence $\left\{\lambda_{n}\right\}_{n \in \mathbf{Z}}$ of complex numbers and construct the sequence of functions $\mathcal{F}=\left\{f_{n}\right\}_{n \in \mathbf{Z}}$ by $f_{n}:[-\gamma, \gamma] \rightarrow \mathbf{C}$, $f_{n}(x)=\frac{1}{\sqrt{2 \gamma}} e^{i \lambda_{n} x}$. We call $\left\{\lambda_{n}\right\}_{n \in \mathbf{Z}}$ a frame sequence if $\mathcal{F}$ is a frame for $L^{2}[-\gamma, \gamma]$, in which case $\mathcal{F}$ is called a Fourier frame. Our problem is the following: given a frame sequence of real numbers $\left\{\lambda_{n}\right\}_{n \in \mathbf{Z}}$ with bounds $A, B$, find a positive constant $L$ such that any sequence of real numbers $\left\{\mu_{n}\right\}_{n \in \mathbf{Z}}$ with $\left|\mu_{n}-\lambda_{n}\right| \leq \delta<L$ is also a frame sequence. An extension of this problem will take into account the complex case.

In the context of an orthonormal Fourier basis $\left(\lambda_{n}=n, \gamma=\pi\right)$ this problem was first considered by Paley and Wiener. By using their stability result, they obtained a first value for $L, L_{1}=\frac{1}{\pi^{2}}$. Later on, Duffin and Eachus in [5] improved this constant to $L_{2}=\frac{\ln 2}{\pi}=0.22 \ldots$. Finally, Kadec in [8] proved that the optimal value of this constant (called the Paley-Wiener constant) is $L_{K}=\frac{1}{4}$ (earlier, Levinson in [10] proved that for $\delta=\frac{1}{4}$ one can perturb the orthonormal Fourier basis to a noncomplete set).

The stability question of Fourier frames was considered by Duffin and Schaeffer in their seminal paper [6]. They used a type (1) inequality with $\mu=0$ and they obtained $L_{D S}=\frac{1}{\gamma} \ln \left[1+\sqrt{\frac{A}{B}}\right.$ ) (see proof of 
Theorem 13, $\S 4.8$ in [12]). This value has been used recently by [3] in a quantization error analysis of Weyl-Heisenberg frame expansions. For $\gamma=\pi$ and $A=B$ one can obtain $L_{D S}=\frac{\ln 2}{\pi}$ which is less than Kadec' estimate. A better estimate for $L$ is given in Theorem 1:

THEOREM 1 Suppose $\left\{\lambda_{n}\right\}_{n \in \mathbf{Z}}$ a frame sequence of real numbers for $L^{2}[-\gamma, \gamma]$ with bounds A, B. Set:

$$
L(\gamma)=\frac{\pi}{4 \gamma}-\frac{1}{\gamma} \arcsin \left(\frac{1}{\sqrt{2}}\left(1-\sqrt{\frac{A}{B}}\right)\right)
$$

Consider the sequence $\left\{\rho_{n}\right\}_{n \in \mathbf{Z}}$ of complex numbers $\rho_{n}=\mu_{n}+i \sigma_{n}$ such that $\sup _{n}\left|\mu_{n}-\lambda_{n}\right|=\delta<L(\gamma)$ and $\sup _{n}\left|\sigma_{n}\right|=M<\infty$. Then the following two conclusions hold true:

1) The sequence $\left\{\rho_{n}\right\}_{n \in \mathbf{Z}}$ is a frame sequence for $L^{2}[-\gamma, \gamma]$;

2) The real sequence $\left\{\mu_{n}\right\}_{n \in \mathbf{Z}}$ is a frame sequence with bounds:

$$
A\left(1-\sqrt{\frac{A}{B}}(1-\cos \gamma \delta+\sin \gamma \delta)\right)^{2}, B(2-\cos \gamma \delta+\sin \gamma \delta)^{2}
$$

We now turn to the statement of our second application. Consider two positive numbers $a_{0}>1$ and $b_{0}>0$ and a function $\Psi \in L^{2}(\mathbf{R})$. We set $H=L^{2}(\mathbf{R}), \mathbf{I}=\mathbf{Z}^{2}$ and define the set of functions $\mathcal{F}_{\Psi ; a_{0} b_{0}}=\left\{\Psi_{m n ; a_{0} b_{0}} \mid(m, n) \in \mathbf{I}\right\}$ by $\Psi_{m n ; a_{0} b_{0}}(x)=a_{0}^{-m / 2} \Psi\left(a_{0}^{-m} x-n b_{0}\right)$. If the set $\mathcal{F}_{\Psi ; a_{0} b_{0}}$ is a frame in $H$ we call it a wavelet frame; likewise, if it is a Riesz basis in $H$ we call it a wavelet Riesz basis. Our problem concerns the behavior of the set $\mathcal{F}_{\Psi ; a b}$ when $a=a_{0}$ and $b$ runs through a neighborhood of $b_{0}$. This problem was first considered by Daubechies and Tchamichian in 1990 for the Meyer orthogonal wavelet basis (see [4]) where $a_{0}=2, b_{0}=1$. They proved that for all $b$ in some nontrivial interval $[1-\varepsilon, 1+\varepsilon]$, the corresponding $\mathcal{F}_{\Psi ; 2 b}$ constituted a Riesz basis. We are going to extend this stability result to a more general class of wavelet Riesz basis. The precise statement is given in Theorem 2:

THEOREM 2 Suppose that the function $\Psi \in L^{2}(\mathbf{R})$ generates a wavelet Riesz basis with bounds $A, B$ for some $a_{0}>1, b_{0}>0$ (i.e. $\mathcal{F}_{\Psi ; a_{0} b_{0}}$ is a Riesz basis with bounds $A, B$ ). Furthermore, let $\hat{\Psi}$, the Fourier transform of $\Psi$, satisfy the following requirement: $\hat{\Psi}$ is of class $\mathcal{C}^{1}$ on $\mathbf{R}$ and both $\hat{\Psi}$ and $\hat{\Psi}^{\prime}$ are bounded by:

$$
|\hat{\Psi}(\xi)|,\left|\hat{\Psi}^{\prime}(\xi)\right| \leq C \frac{|\xi|^{\alpha}}{(1+|\xi|)^{\gamma}} \quad, \quad \forall \xi \in \mathbf{R}
$$

for some $C>0$ and $\gamma>1+\alpha>1$. Then there exists an $\varepsilon>0$ such that for any $b$ with $\left|b-b_{0}\right|<\varepsilon$, the set $\mathcal{F}_{\Psi ; a_{0} b}$ is a Riesz basis.

\section{Proof of the Theorems}

Proof of Theorem 1

By Theorem II from [6] (see also Theorem 14, $§ 4.8$ in [12]) we need to prove Theorem 1 only for real sequences $\rho_{n}=\mu_{n}$. On the other hand, if we scale the sequence we can reduce the problem to the case $\gamma=\pi$. Indeed, if $\left\{\lambda_{n}\right\}_{n \in \mathbf{Z}}$ is a frame sequence for $L^{2}[-\gamma, \gamma]$ then $\left\{\lambda_{n}^{\prime}=\frac{\gamma}{\pi} \lambda_{n}\right\}_{n \in \mathbf{Z}}$ is a frame sequence for $L^{2}[-\pi, \pi]$ with the same bounds (in the former case $f_{n}(x)=\frac{1}{\sqrt{2 \gamma}} e^{i \lambda_{n} x}$, in the latter $f_{n}^{\prime}(x)=\frac{1}{\sqrt{2 \pi}} e^{i \lambda_{n}^{\prime} x}$ ). Thus $L(\gamma)=\frac{\pi}{\gamma} L(\pi)$ and we have to prove:

$$
L(\pi)=\frac{1}{4}-\frac{1}{\pi} \arcsin \left(\frac{1}{\sqrt{2}}\left(1-\sqrt{\frac{A}{B}}\right)\right)
$$


Observe that this is consistent also with the frame bounds since $\gamma \delta=\pi \delta^{\prime}$.

To prove (6), we shall use Kadec' estimations from his theorem and then the Type 2 form of the Stability Theorem with $\lambda=0$. Let $N \in \mathbf{N}$ and $c_{n} \in \mathbf{C}, n \in I_{N}$ be arbitrary. Set $\delta_{n}=\mu_{n}-\lambda_{n}$. We obtain:

$$
U=\left\|\sum_{n \in I_{N}} c_{n}\left(\frac{1}{\sqrt{2 \pi}} e^{i \lambda_{n} x}-\frac{1}{\sqrt{2 \pi}} e^{i \mu_{n} x}\right)\right\|=\frac{1}{\sqrt{2 \pi}}\left\|\sum_{n \in I_{N}} c_{n} e^{i \lambda_{n} x}\left(1-e^{i \delta_{n} x}\right)\right\|
$$

By expanding $1-e^{i \delta_{n} x}$ into a Fourier series relative to the orthogonal system $\left\{1, \cos \nu x, \sin \left(\nu-\frac{1}{2}\right) x\right\}$, $\nu=1,2, \ldots$ we obtain:

$$
1-e^{i \delta_{n} x}=\left(1-\frac{\sin \pi \delta_{n}}{\pi \delta_{n}}\right)+\sum_{\nu=1}^{\infty} \frac{(-1)^{\nu} 2 \delta_{n} \sin \pi \delta_{n}}{\pi\left(\nu^{2}-\delta_{n}{ }^{2}\right)} \cos (\nu x)+i \sum_{\nu=1}^{\infty} \frac{(-1)^{\nu} 2 \delta_{n} \cos \pi \delta_{n}}{\pi\left(\left(\nu-\frac{1}{2}\right)^{2}-\delta_{n}{ }^{2}\right)} \sin \left(\left(\nu-\frac{1}{2}\right) x\right)
$$

We plug (8) into (7), we change the order of summation, we use the triangle inequality and then we use the bounds $\|\cos (\nu x) \varphi(x)\| \leq\|\varphi\|$ and $\left\|\sin \left(\left(\nu-\frac{1}{2}\right) x\right) \varphi(x)\right\| \leq\|\varphi\|$. We obtain:

$$
U \leq\left\|\sum_{n \in I_{N}}\left(1-\frac{\sin \pi \delta_{n}}{\pi \delta_{n}}\right) c_{n} e^{i \lambda_{n} x}\right\|+\sum_{\nu=1}^{\infty}\left(\left\|\sum_{n \in I_{N}} \frac{2 \delta_{n} \sin \pi \delta_{n}}{\pi\left(\nu^{2}-\delta_{n}^{2}\right)} c_{n} e^{i \lambda_{n} x}\right\|+\left\|\sum_{n \in I_{N}} \frac{2 \delta_{n} \cos \pi \delta_{n}}{\pi\left(\left(\nu-\frac{1}{2}\right)^{2}-\delta_{n}^{2}\right)} c_{n} e^{i \lambda_{n} x}\right\|\right)
$$

Now we use that $\left\{\lambda_{n}\right\}_{n \in \mathbf{Z}}$ is a frame sequence with upper bound $B$. Therefore each norm can be bounded as:

$$
\left\|\sum_{n \in I_{N}} a_{n} c_{n} e^{i \lambda_{n} x}\right\| \leq \sqrt{B}\left\|\left\{a_{n} c_{n}\right\}\right\| \leq \sqrt{B} \sup _{n}\left|a_{n}\right|\left\|\left\{c_{n}\right\}\right\|
$$

and we have:

$$
\begin{aligned}
\left|1-\frac{\sin \pi \delta_{n}}{\pi \delta_{n}}\right| & \leq 1-\frac{\sin \pi \delta}{\pi \delta} \\
\left|\frac{2 \delta_{n} \sin \pi \delta_{n}}{\pi\left(\nu^{2}-\delta_{n}{ }^{2}\right)}\right| & \leq \frac{2 \delta \sin \pi \delta}{\pi\left(\nu^{2}-\delta^{2}\right)} \\
\left|\frac{2 \delta_{n} \cos \pi \delta_{n}}{\pi\left(\left(\nu-\frac{1}{2}\right)^{2}-\delta_{n}{ }^{2}\right)}\right| & \leq \frac{2 \delta \cos \pi \delta}{\pi\left(\left(\nu-\frac{1}{2}\right)^{2}-\delta^{2}\right)}
\end{aligned}
$$

(the last inequality holds because $\delta<\frac{1}{4}$ ) and thus:

$$
U \leq \sqrt{B}\left(R e\left(1-e^{i \pi \delta}\right)-\operatorname{Im}\left(1-e^{i \pi \delta}\right)\right)\left(\sum_{n \in I_{N}}\left|c_{n}\right|^{2}\right)^{1 / 2}
$$

or:

$$
U \leq \sqrt{B}(1-\cos \pi \delta+\sin \pi \delta)\left(\sum_{n \in I_{N}}\left|c_{n}\right|^{2}\right)^{1 / 2}
$$

Now we can apply the Stability Theorem (Type 2) with $\lambda=0$ and $\mu=\sqrt{B}(1-\cos \pi \delta+\sin \pi \delta)$. The condition of that theorem turns into $\mu<\sqrt{A}$ or $1-\cos \pi \delta+\sin \pi \delta<\sqrt{\frac{A}{B}}$ and then, by a little trigonometry we get:

$$
\delta<L=\frac{1}{4}-\frac{1}{\pi} \arcsin \left(\frac{1}{\sqrt{2}}\left(1-\sqrt{\frac{A}{B}}\right)\right)
$$

The frame bounds for $\left\{\mu_{n}\right\}_{n \in \mathbf{Z}}$ come from $A\left(1-\frac{\mu}{\sqrt{A}}\right)^{2}$ and $B\left(1+\frac{\mu}{\sqrt{B}}\right)^{2}$. This ends the proof. $\diamond$

To prove Theorem 2, we shall use the Type 1 criterion of stability together with an upper bound estimation given by Daubechies in [4] Theorem 2.7,2.8. This estimate reads as follows: Consider $f \in$ 
$L^{2}(\mathbf{R})$ and, for some $a>1, b>0$ construct the set of functions $\mathcal{F}_{f ; a b}$ as before. Then, a sufficient condition for $\mathcal{F}_{f ; a b}$ to be a Bessel set for $L^{2}(\mathbf{R})$ is that:

$$
B:=\frac{2 \pi}{b}\left\{\sup _{1 \leq|\xi| \leq a} \sum_{m \in \mathbf{Z}}\left|\hat{f}\left(a^{m} \xi\right)\right|^{2}+2 \sum_{k=1}^{\infty}\left(\beta\left(\frac{2 \pi}{b} k\right) \beta\left(-\frac{2 \pi}{b} k\right)\right)^{1 / 2}\right\}<\infty
$$

where $\beta(s)=\sup _{1 \leq|\xi| \leq a} \sum_{m \in \mathbf{Z}}\left|\hat{f}\left(a^{m} \xi\right)\right| \cdot\left|\hat{f}\left(a^{m} \xi+s\right)\right|$. Moreover, the constant $B$ in (9) is an upper bound of the Bessel sequence. A sufficient condition for (9) to hold for is that the function $\hat{f}(\xi)$ has good decay, for instance $|\hat{f}(\xi)| \leq C \frac{|\xi|^{\alpha}}{(1+|\xi|)^{\gamma}}$, for some $\gamma>1+\alpha>1$ and $C>0$.

Now we return to the proof of theorem:

Proof of Theorem 2

Consider $\Psi$ and $a_{0}>1, b_{0}>0$ and $b>0$ as in hypothesis and denote by $U_{b}: L^{2}(\mathbf{R}) \rightarrow L^{2}(\mathbf{R})$ the unitary operator $\left(U_{b} f\right)(x)=\sqrt{\frac{b}{b_{0}}} f\left(\frac{b}{b_{0}} x\right)$. We define $\Phi=U_{b} \Psi$, or more specific $\Phi(x)=\sqrt{\frac{b}{b_{0}}} \Psi\left(\frac{b}{b_{o}} x\right)$. One can easily check that $U_{b} \Psi_{m n ; a_{0} b}=\Phi_{m n ; a_{0} b_{0}}$, therefore $U_{b}$ maps $\mathcal{F}_{\Psi ; a_{0} b}$ into $\mathcal{F}_{\Phi ; a_{0} b_{0}}$ unitarily. Thus $\mathcal{F}_{\Psi ; a_{0} b}$ is a Riesz basis (frame) if and only if $\mathcal{F}_{\Phi ; a_{0} b_{0}}$ is a Riesz basis (frame). Moreover, they have the same bounds. In order to prove that $\mathcal{F}_{\Psi ; a_{0} b}$ is a Riesz basis, we show that $\mathcal{F}_{\Phi ; a_{0} b_{0}}$ is a Riesz basis by comparing it with $\mathcal{F}_{\Psi ; a_{0} b_{0}}$. We note that:

$$
\Psi_{m n ; a_{0} b_{0}}-\Phi_{m n ; a_{0} b_{0}}=(\Psi-\Phi)_{m n ; a_{0} b_{0}}
$$

Therefore the condition (1) with $\lambda=0$ is equivalent with the condition $\mathcal{F}_{\Psi-\Phi ; a_{0} b_{0}}$ to be a Bessel set with upper bound less than $A$, the lower frame bound of the Riesz basis $\mathcal{F}_{\Psi ; a_{0} b_{0}}$.

Let us denote by $B_{\alpha, \gamma}$ the constant $B$ given by $(9)$ for $\hat{f}(\xi)=\frac{\mid \xi^{\alpha}}{(1+|\xi|)^{\gamma}}$. It is simple to check that $|\hat{\Psi}(\xi)-\hat{\Phi}(\xi)| \leq C_{b} \frac{|\xi|^{\alpha}}{(1+|\xi|)^{\gamma}}$. Therefore an upper bound for the Bessel set $\mathcal{F}_{\Psi-\Phi ; a_{0} b_{0}}$ is given by $C_{b} B_{\alpha, \gamma}$. On the other hand, using the Ascoli-Arzelá lemma and the hypotheses on $\hat{\Psi}(\xi)$ and $\hat{\Psi}^{\prime}(\xi)$ we obtain that $g_{b}(\xi)=\frac{\left(1+\left.|\xi|\right|^{\gamma}\right.}{|\xi|^{\alpha}} \hat{\Phi}(\xi)$ converges uniformly to $g_{b_{0}}(\xi)=\frac{(1+|\xi|)^{\gamma}}{|\xi|^{\alpha}} \hat{\Psi}(\xi)$ as $b \rightarrow b_{0}$. Thus we may choose $C_{b}$ to depend continuously on $b$ around $b_{0}$ and $C_{b_{0}}=0$. Then, for some neighborhood of $b_{0}$ for which $C_{b} B_{\alpha \gamma}<A$ we may set $\mu=\sqrt{C_{b} B_{\alpha \gamma}}$ in (1) and we obtain that $\mathcal{F}_{\Phi ; a_{0} b_{0}}$ is a Riesz basis. Now the proof is complete.

\section{Conclusions}

The first theorem proved in this paper gives a better stability bound of Fourier frames than known previously in literature. This result covers also Kadec' $\frac{1}{4}$ theorem and extends its conclusion to Fourier tight frames. One can check that:

$$
\frac{\pi}{4 \gamma}-\frac{1}{\gamma} \arcsin \left(\frac{1}{\sqrt{2}}\left(1-\sqrt{\frac{A}{B}}\right)\right)>\frac{1}{\gamma} \ln \left(1+\sqrt{\frac{A}{B}}\right)
$$

which means that the stability margin given by theorem 1 is larger than $L_{D S}$.

The second result shows that wavelets Riesz bases are stable under translation parameter perturbations provided some mild regularity and decaying conditions are satisfied by the wavelet. In particular it applies also to Meyer's orthogonal wavelet basis and thus extends a previously known result.

\section{Acknowledgements}

I would like to thank Prof. Ingrid Daubechies who introduced me in this field and spent a lot of time to teach me many things of analysis. I also thank Prof. Charles Fefferman for a very useful discussion and Prof. Zalik for a copy of [7] which I received it during the final preparation of this paper. 


\section{References}

[1] O.Christensen, A Paley-Wiener Theorem for Frames, Proc.Amer.Math.Soc. 123 (1995), 21992202

[2] O.Christensen, C.Heil, Perturbations of Banach Frames and Atomic Decompositions, to appear in Math.Nach. or http@tyche.mat.univie.ac.at

[3] Z.Cvetković, M.Vetterli, Error Analysis in Oversampled A/D Conversion and Quantization of Weyl-Heisenberg Frame Expansions, Mem.No. UCB/ERL M95/48, May (1995)

[4] I.Daubechies, The Wavelet Transform, Time-Frequency Localizatin and Signal Analysis, IEEE Trans.Inform. Theory, no.5, 36 (1990), 961-1005

[5] R.J.Duffin, J.J.Eachus, Some Notes on an Expansion Theorem of Paley and Wiener, Bull.Amer.Math.Soc., 48 (1942), 850-855

[6] R.J.Duffin, A.C.Schaeffer, A Class of Nonharmonic Fourier Series, Trans.of AMS, 72, (1952), $341-366$

[7] S.J.Favier, R.A.Zalik, On the stability of frames and Riesz bases, Appl.Comp.Harmon.Annal., no.2, 2 (1995), 160-173

[8] M.I.Kadec, The Exact Value of the Paley-Wiener Constant, Sov.Math.Doklady, no.2, 5, (1964), $559-561$

[9] T.Kato, Perturbation Theory for Linear Operators, Springer-Verlag (1976)

[10] N.Levinson, Gap and Density Theorems, AMS Coll. Public., 26, (1940)

[11] R.E.A.C.Paley, N.Wiener, Fourier Transforms in the Complex Domain, AMS Colloq.Publ., vol.19, AMS, Providence R.I. (1934), reprint 1960

[12] R.M.Young, An Introduction to Nonharmonic Fourier Series, Academic Press (1980) 